\title{
Shape of the Landau subbands in disordered graphene
}

\author{
W. Zhu, ${ }^{1,2}$ H. Y. Yuan, ${ }^{2}$ Q. W. Shi, ${ }^{1}$ J. G. Hou, ${ }^{1}$ and X. R. Wang ${ }^{2,3, *}$ \\ ${ }^{1}$ Hefei National Laboratory for Physical Sciences at Microscale, University of Science and Technology of China, \\ Hefei, People's Republic of China \\ ${ }^{2}$ Department of Physics, The Hong Kong University of Science and Technology, Clear Water Bay, Kowloon, Hong Kong \\ ${ }^{3}$ School of Physics, Shandong University, Jinan, People's Republic of China
}

(Received 2 March 2011; published 18 April 2011)

\begin{abstract}
The question of why different experiments obtain different shapes of Landau subbands of two-dimensional electron gases (2DEG's) is investigated. In particular, we consider disordered graphene within the tight-binding formulation in a high magnetic field and in the presence of impurity potentials of a finite interaction range. It is found that the shape of the total density of states (DOS) of Landau subbands is well described by a Gaussian function, while the shape of the local density of states (LDOS) can be fitted to either a simple Lorentzian function or a sum of several Lorentzian functions. This finding explains well why the experiments that measure the DOS of a Landau subband appear to be Gaussian, and those experiments that are sensitive to the LDOS can only be fitted well by Lorentzian functions. Thus, our results provide a natural explanation to this long-standing puzzle involving 2DEG's in the quantum Hall regime.
\end{abstract}

DOI: 10.1103/PhysRevB.83.153408

PACS number(s): 81.05.ue, 71.55.-i, 71.23.-k

Graphene has attracted a lot of attention due to its remarkable properties and its potential applications in nanoelectronics. ${ }^{1}$ Its electronic properties are mainly due to its two-dimensional nature and the fact that the low-energy excitations are governed by the massless Dirac equation, ${ }^{2}$ confirmed by the interesting, uneven Landau level (LL) distribution that varies with both the square root of the magnetic field $B$ and the LL index $n: E_{n}=\operatorname{sgn}(n) \sqrt{2 e \hbar v_{F}^{2}|n| B}$, where $v_{F}, e$, and $\hbar$ are the Fermi velocity, electron charge, and the Planck constant, respectively. This emblematic LL spectrum and its characteristic zero-energy state $(n=0)$ are directly related to the anomalous quantum Hall effect (QHE) in graphene. ${ }^{3}$ Many efforts have been made to determine the electronic properties of the disorder-broadened Landau subbands (LS's). However, the shape of both the total density of states (DOS) and the local DOS (LDOS) of an LS of graphene is a controversial issue in the literature. Historically, it has generally been believed that the shape of the DOS of LS's in a conventional two-dimensional electron gas (2DEG) is Gaussian-like, ${ }^{4,5}$ but experiments seem to present a different picture. Initially, this consensus was supported by careful measurements of specific heat, ${ }^{6}$ magnetization, ${ }^{7}$ and magnetocapacitance ${ }^{8}$ on $\mathrm{GaAs}-\mathrm{Ga}_{x} \mathrm{Al}_{1-x}$ As heterostructures. However, a recent scanning-tunneling-microscopy-scanningtunneling-spectroscopy (STM-STS) measurement on conventional 2DEG's of the $n$-InSb(110) [or $n$-InAs(110)] surface, ${ }^{9}$ as well as the cyclotron resonance (CR) absorption line shape on conventional 2DEG's, ${ }^{10,11}$ observed a Lorentzian shape of an LS. The situation for disordered graphene is similar. All $\mathrm{CR}^{13-15}$ and STM-STS ${ }^{16-19}$ measurements suggested clearly a Lorentzian shape for an LS. However, recent magnetocapacitance experiments produced conflicting results: In one experiment, ${ }^{20}$ an LS could be fitted well by the Lorentzian functions, while another experiment ${ }^{21}$ observed a Gaussian shape for an $n=0 \mathrm{LS}$. On the theoretical side, the DOS of an LS of graphene is initially found to be Gaussian-like. ${ }^{22-24}$ Champel et al. ${ }^{25}$ found from a recent Green's function calculation that the LDOS's of LS's are also Gaussian. Thus, the theories do not explain the observed Lorentzian shape of the DOS or the LDOS. This long-standing question regarding Lorentzian or Gaussian LS's is the focus of this study.

In this paper, we use the Lanczos recursion method to numerically compute both the DOS and the LDOS of disordered graphene in high magnetic field and in the presence of a random potential of a finite interaction range. We find that the shape of the DOS of the disorder-broadened LS's is Gaussian regardless of their potential range. However, the shape of the LDOS of an LS can be fitted to either a simple Lorentzian function or the sum of several Lorentzian functions. Therefore, our results suggest that all local measurements should be described by Lorentzian functions, while global measurements such as specific heat and magnetocapacitance are Gaussian.

There are two types of experiments. One is sensitive to global electronic properties. For example, the specific heat ${ }^{6}$ and magnetocapacitance ${ }^{8}$ are directly related to the DOS of a sample. The other type of experiment, such as STM, probes the local energy level or the LDOS. ${ }^{9,16-19,26}$ The majority of experiments on both graphene and conventional 2DEG's suggest that Gaussian LS's appear in global measurements while Lorentzian LS's appear in local measurements. This is a plausible conjecture because almost all electronic states in two-dimensional QHE systems are localized except the one at the center of each LS (necessary for observing QHE). By definition, there is no correlation among different localized states. Thus, the DOS is expected to be Gaussian since it involves the distribution of a group of independent states that should obey a large-number theorem. On the other hand, the LDOS is dominated by one or a few states from an LS, and the natural line shape of a single quantum level is known to be Lorentzian. To test this conjecture, one needs to calculate both the DOS and the LDOS of a 2DEG in the QHE regime. We consider graphene in the presence of a high field and disorders below.

Low-energy excitations of graphene come from the $\pi$ electrons that can be modeled by a tight-binding Hamiltonian 
on a honeycomb lattice,

$$
H=\sum_{\langle i j\rangle} t e^{i \phi_{i j}}|i\rangle\langle j|+\text { H.c. }+\sum_{i} \varepsilon_{i}|i\rangle\langle i|,
$$

where $|i\rangle$ denotes the $\pi$-electron state on site $i . t=-2.7 \mathrm{eV}$ is the hopping energy between two nearest-neighbor sites. The magnetic field is introduced by Peierls' substitution in the phase of the hopping parameter ${ }^{27} \phi_{i j}=2 \pi e / h \int_{i}^{j} \mathbf{A} \cdot d \mathbf{l}$, where $\mathbf{A}$ is the vector potential. The disorders are introduced through random on-site energy $\varepsilon_{i}=V\left(\mathbf{r}_{i}\right)$ in the second term of Eq. (1). In our model, we assume that the random potential is generated by $N_{i}$ randomly distributed impurities whose potential takes a Gaussian form with an interaction range of $d,{ }^{28}$

$$
V\left(\mathbf{r}_{i}\right)=\sum_{m=1}^{N_{i}} \frac{u_{m}}{u_{0}} \exp \left(-\frac{\left|\mathbf{r}_{i}-\mathbf{r}_{m}\right|^{2}}{d^{2}}\right),
$$

where $u_{m}$ is a random number uniformly distributed in $[-W, W] . \quad u_{0}$ is a normalization constant that guarantees the impurity potential satisfying the condition $\sum_{\mathbf{r}_{\mathbf{i}}} \exp \left(-\mathbf{r}_{i}^{2} / d^{2}\right)=u_{0} \cdot{ }^{29}$ For a lattice of a total of $N$ lattice sites, $n_{\text {imp }}=N_{i} / N$ is the impurity concentration. It is known that the extrinsic spatial disorder is crucial for electronic structures and transport properties of graphene. ${ }^{2}$ In what follows, we will discuss the impurities potential with a very short interaction range $(d<a)$ ( $a$ is a lattice constant) and an interaction range bigger than the lattice constant $(d>a)$. Since the spin degrees of freedom contribute only a tiny energy through the Zeeman effect, and will be irrelevant to the discussion below, we neglect the spin index in our model.

The LDOS of the Hamiltonian [Eq. (1)] can be evaluated by $\rho\left(\mathbf{r}_{i}, E\right)=-\operatorname{Im}\left\langle i\left|\frac{1}{E-H+i v}\right| i\right\rangle / \pi$, where $\nu$ is an infinitesimal positive number. An accurate LDOS is numerically obtainable by using the well-developed Lanczos recursive method. ${ }^{24}$ The DOS can also be obtained by averaging over all LDOS's and/or through an ensemble average. In this approach, a small artificial cutoff energy $\epsilon=1 \mathrm{meV}$ is introduced to simulate the infinitesimal imaginary energy $v,{ }^{31,32}$ which will lead to a small width of LL's in clean graphene. To reduce the finite-size effects, a very large lattice of more than one million sites $\left(N>10^{6}\right)$ is used. In the calculations, the periodic boundary conditions are imposed.

In the absence of disorders, the system has a $\delta$-like LL spectrum as shown in Fig. 1(a). The seemingly nonzero width is due to cutoff energy $\epsilon$, which may describe electron-phonon or electron-electron interactions in reality. ${ }^{24}$ Also, there are no differences in the shape of the DOS and the LDOS. These natural broadened spectra follow the Lorentzian distribution as expected. In the presence of disorders, for the total averaged DOS shown in Fig. 1(b), the highly degenerate LL's are broadened into LS's due to the disorder effects. ${ }^{24}$ Similar to the DOS in Fig. 1(b), the LDOS in Fig. 1(c) reveals also the LL's spectrum, but their shapes are asymmetric and much sharper in comparison with DOS. In what follows, we will focus on the shape of the DOS and the LDOS of LS's, especially for $n=0 \mathrm{LS}$.

To have a better knowledge about the random potential used in our calculations, Fig. 2(a) is the contour plot of a typical random potential generated by randomly distributed impurities of interaction range $d=0.1 a$, much smaller than the lattice constant, so that each impurity generates only a

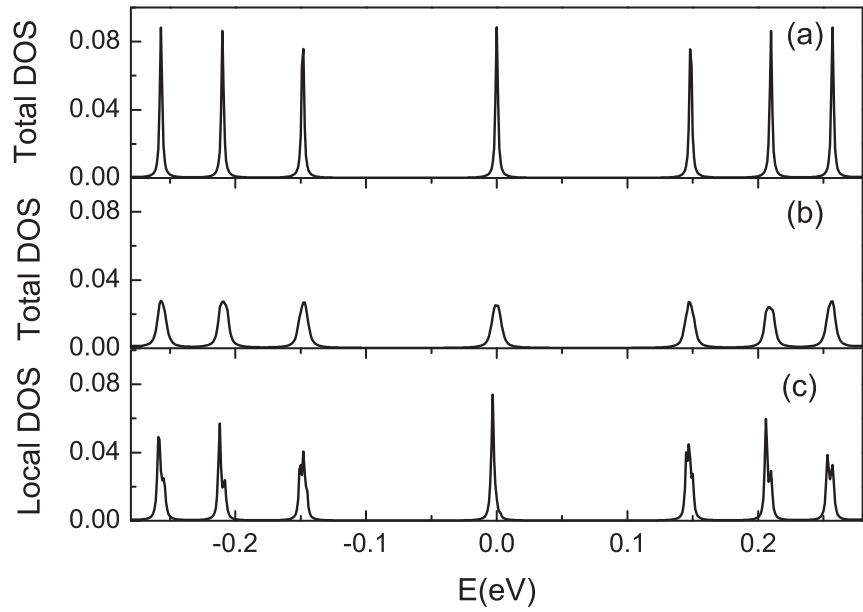

FIG. 1. (a) DOS-LDOS of LL's of clean graphene. The effects of the impurity potential of finite range $d=0.1 a$ on (b) the DOS and (c) the LDOS of LS's of graphene for $B=22.0 \mathrm{~T}$. The illustration of the impurity potential is shown in Fig. 2(a).

random on-site energy. This local potential feature can indeed been seen from the dot structure in the figure. In comparison, Fig. 3(a) is a similar plot for the impurity potential of the interaction range $d=5 a$. The contours of the generated random potential have a loop structure. Figure 2(b) is the total averaged DOS of an $n=0 \mathrm{LS}$ in the random potential of Fig. 2(a). The DOS can be well fitted by a Gaussian function, $\frac{A}{\Gamma_{G} \sqrt{\pi / 2}} e^{-2 E^{2} / \Gamma_{G}^{2}}$, with a broadening width $\Gamma_{G}=6.0 \mathrm{meV}$, in agreement with an early study ${ }^{24}$ where $\Gamma_{G}$ is proportional to the random potential deviation and the square root of magnetic field $B$. Figures 2(c) and 2(d) are two typical LDOS's of an
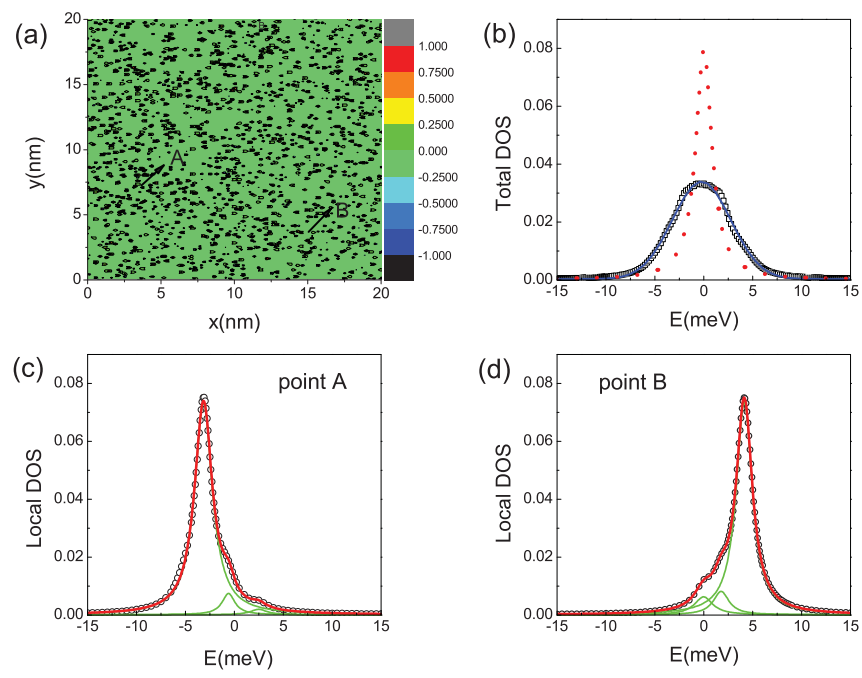

FIG. 2. (Color online) (a) Contour plot of a randomly generated impurity potential of finite range $d=0.1 a$. The impurity concentration and parameter are $n_{\text {imp }}=5.6 \%$ and $W=1.0 \mathrm{eV}$, respectively. (b) Total averaged DOS of $n=0$ LS (open square) in the random potential of (a). The heavy blue line is the fitted Gaussian curve. For comparison, the DOS of clean graphene is also shown (red dotted line). The LDOS of $n=0 \mathrm{LS}$ at sites A (c) and B (d) in (a) are denoted by open circles. The heavy red lines are the best fit to the sum of three Lorentzian functions (thin green lines are the individual Lorentzian curves). All the results are calculated in $B=22.0 \mathrm{~T}$. 

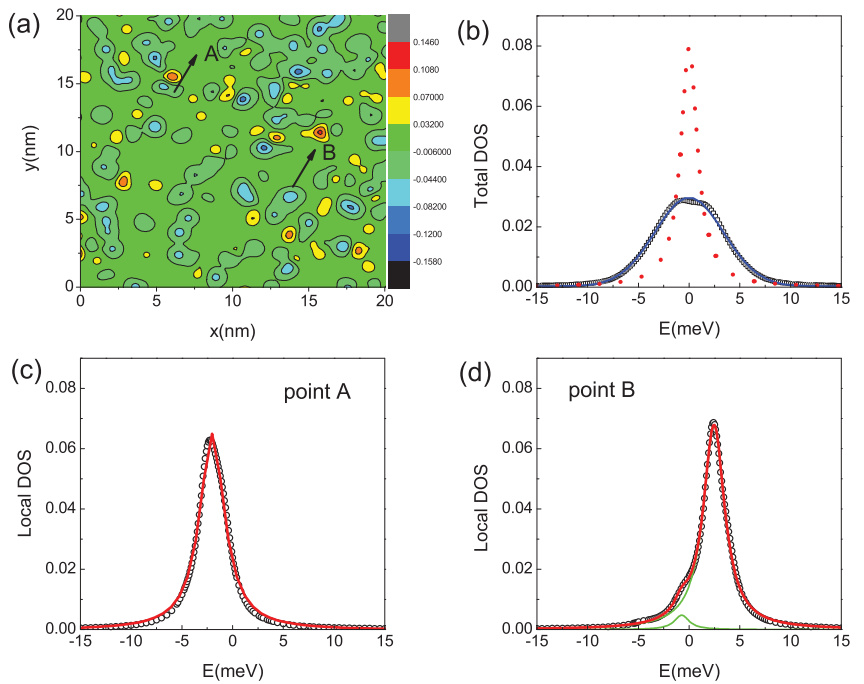

FIG. 3. (Color online) (a) Contour plot of a randomly generated impurity potential of finite range $d=5.0 a$. The impurity concentration and parameter are $n_{\text {imp }}=1.0 \%$ and $W=4.0 \mathrm{eV}$. (b) The total averaged DOS of $n=0$ LS (open square) fits well to the Gaussian function (heavy blue line). The DOS of clean graphene (red dotted line) is also plotted for a comparison. (c) Typical LDOS (open circle) at site A of (a). Only one Lorentzian curve (heavy red line) is used to fit the data. (d) Typical LDOS (open circle) at site B. The heavy red line is a fit to a sum of two Lorentzian functions (thin green lines).

$n=0 \mathrm{LS}$ at sites A and B of Fig. 2(a), respectively. The peaks are clearly shifted away (positive for A and negative for B) from the Dirac point due to the random potential there. Two shoulders indicate that the LDOS's are mainly from three states. Thus, it is natural to use the sum of three Lorentzian functions $\sum_{i=1}^{3} \frac{2 A_{i}}{\pi} \frac{\Gamma_{i}}{4\left(E-E_{i}\right)^{2}+\Gamma_{i}^{2}}$ to fit the numerical data. The LDOS at $\mathrm{A}$ can be fitted by one dominating Lorentzian function with $A_{1}=0.254, \Gamma_{1}=2.2 \mathrm{meV}, E_{1}=-3.16 \mathrm{meV}$ and two minor Lorentzian contributions with parameters $A_{2}=0.018, \quad \Gamma_{2}=1.85 \mathrm{meV}, \quad E_{2}=-0.6 \mathrm{meV} \quad$ and $A_{3}=0.006, \Gamma_{3}=2.0 \mathrm{meV}, E_{3}=2.5 \mathrm{meV}$. The fact that $\Gamma_{i}$ is bigger than the natural width in Fig. 1(a) suggests that the widths of the LDOS are due to the random potential. The good Lorentzian description for the LDOS applies also for other parts of the sample. Figure 2(d) shows that the LDOS at point $\mathrm{B}$ can be fitted by one dominating Lorentzian function with $A_{1}=0.231, \Gamma_{1}=2.0 \mathrm{meV}, E_{1}=4.13 \mathrm{meV}$ and two minor Lorentzian functions with $A_{2}=0.024, \Gamma_{2}=1.95 \mathrm{meV}, E_{2}=$ $1.8 \mathrm{meV}$ and $A_{3}=0.022, \quad \Gamma_{3}=2.26 \mathrm{meV}, \quad E_{3}=$ $-0.02 \mathrm{meV}$. These results suggest that the LDOS's at the sites are mainly from a few states in the zero LS, since the natural shape of each state is Lorentzian and the LDOS is

$$
\rho\left(\mathbf{r}_{j}, E\right)=\frac{1}{\pi} \sum_{i} \frac{\Gamma_{i} / 2}{\left(E-E_{i}\right)^{2}+\left(\Gamma_{i} / 2\right)^{2}}\left|\psi_{i}\left(\mathbf{r}_{j}\right)\right|^{2},
$$

where $E_{i}$ is the energy of the state $\psi_{i}(\mathbf{r})$ and $\Gamma_{i}$ is the level broadening width due to the randomness. Of course, this is consistent with the fact that all states must be localized except one in each LS in order to observe a QHE.

To show that the above results do not depend on the details of the random potential, we have computed the same quantities for other potentials (with various impurity concentrations and interaction ranges $0.1 a<d<40 a$ ), and we obtained the same results. For example, Fig. 3 shows the results for the impurities potential of the interaction range $d=5.0 a$. The total averaged DOS $n=0 \mathrm{LS}$ (open squares) follows again the Gaussian distribution (blue line with $\Gamma_{G}=7.0 \mathrm{meV}$ ), as shown in Fig. 3(b). Figure 3(c) is the LDOS at site A in Fig. 3(a). The curve is approximately symmetric and no obvious shoulder exists so that we use a single Lorentzian to fit the numerical data (open circles). The fit with $\Gamma_{1}=2.96 \mathrm{meV}$ and $E_{1}=-2.0 \mathrm{meV}$ is excellent. The LDOS [Fig. 3(d)] at site B in Fig. 3(a) is not symmetric around its peak and a shoulder is clearly present so that two states contribute to the LDOS. Indeed, the data [open circles in Fig. 3(d)] can be well described by a dominating Lorentzian function with $A_{1}=$ $0.273, \Gamma_{1}=2.6 \mathrm{meV}, E_{1}=2.5 \mathrm{meV}$ and a minor Lorentzian function with $A_{2}=0.013, \Gamma_{2}=1.76 \mathrm{meV}, E_{1}=-0.7 \mathrm{meV}$. These LDOS's can again be understood from Eq. (3).

Our results provide a natural explanation to the puzzle of why the shapes of LS's can be either Gaussian or Lorentzian, depending on whether an experiment measures the DOS or the LDOS. There is no question that an STM-STS experiment ${ }^{16,17,19}$ probes the LDOS. That is why all STM results yield Lorentzian DOS's whose peak position varies from one location to another randomly, as was observed in experiment. ${ }^{16}$ The cyclotron absorption between two LS's measures the LDOS if the far-infrared light spot size is comparable with the localization length of the localized electron states that they probe. This may be true for a 2DEG in the QHE regime because the electron localization length near the subband center is usually large. This may explain why the cyclotron absorption curve is Lorentzian rather than Gaussian for the DOS of LS's.

We would like to make several remarks before ending this paper. (i) Although the study is on disordered graphene, the results on the shapes of the DOS and the LDOS should be valid for conventional 2DEG's in the QHE regime. This is because the interpretation of our numerical data can also be applied to a conventional 2DEG. Of course, it should still be interesting to double check the results by carrying out a similar investigation for conventional 2DEG's. (ii) The electron-electron interaction is neglected in this study. The interaction may modify the peak positions and peak widths of LS's since it introduces extra scattering channels. It should be interesting to investigate how the interaction modifies the DOS and the LDOS of LS's of graphene. (iii) Our focus is on the shape of an $n=0$ LS. Although the results are expected to be applicable to other subbands, one should anticipate that a small asymmetric distortion may occur in higher LS's in a finite magnetic field, as explained in Ref. 24. (iv) Although most experiments are consistent with our theory, there are also reports ${ }^{20,21,25}$ that deviate, showing that the shape of the DOS and the LDOS of LS's of two-dimensional systems may be more complicated and needs further investigations. For example, as explanation should be sought as to why those few exceptional cases can deviate from the plausible picture presented here.

In conclusion, the DOS and the LDOS of disordered graphene in the QHE regime are evaluated numerically. It is found that the DOS and the LDOS of LS's can be described by Gaussian and Lorentzian functions, respectively. Both 
shapes are robust against the interaction range of an impurity potential. This finding provides a natural explanation to the long-standing puzzle of why the local probes of the DOS usually find a Lorentzian shape of an LS while the global probes of the DOS detect a Gaussian shape.
This work is supported by Hong Kong RGC Grants (No. 603508, No. 604109, HKU10/CRF/08- HKUST17/CRF/08, and RPC10SC05); the NNSF of China (No. 10974187); NKBRP of China (No. 2006CB922005); and KIP of the Chinese Academy of Sciences (No. KJCX2-YW-W22). *phxwan@ust.hk

${ }^{1}$ K. S. Novoselov, A. K. Geim, S. V. Morozov, D. Jiang, Y. Zhang, S. V. Dubonos, I. V. Grigorieva, and A. A. Firsov, Science 306, 666 (2004).

${ }^{2}$ A. H. Castro Neto, F. Guinea, N. M. R. Peres, K. S. Novoselov, and A. K. Geim, Rev. Mod. Phys. 81, 109 (2009).

${ }^{3}$ K. S. Novoselov, A. K. Geim, S. V. Morozov, D. Jiang, M. I. Katsnelson, I. V. Grigorieva, S. V. Dubonos, and A. A. Firsov, Nature (London) 438, 197 (2005); Y. Zhang, Y. -W. Tan, H. L. Stormer, and P. Kim, ibid. 438, 201 (2005).

${ }^{4}$ The Quantum Hall Effect, edited by R. E. Prange and S. M. Girvin (Springer, New York, 1987).

${ }^{5}$ The Physics of the Two-Dimensional Electron Gas, edited by J. T. Devreese and F. M. Peeters (Plenum, New York, 1987).

${ }^{6}$ E. Gornik, R. Lassnig, G. Strasser, H. L. Stormer, A. C. Gossard, and W. Wiegmann, Phys. Rev. Lett. 54, 1820 (1985).

${ }^{7}$ J. P. Eisenstein, H. L. Stormer, V. Narayanamurti, A. Y. Cho, A. C. Gossard, and C. W. Tu, Phys. Rev. Lett. 55, 875 (1985).

${ }^{8}$ V. Mosser, D. Weiss, K. v. Klitzing, K. Ploog, and G. Weimann, Solid State Commun. 58, 5 (1986).

${ }^{9}$ M. Morgenstern, J. Klijn, Chr. Meyer, M. Getzlaff, R. Adelung, R. A. Romer, K. Rossnagel, L. Kipp, M. Skibowski, and R. Wiesendanger, Phys. Rev. Lett. 89, 136806 (2002); K. Hashimoto, C. Sohrmann, J. Wiebe, T. Inaoka, F. Meier, Y. Hirayama, R. A. Romer, R. Wiesendanger, and M. Morgenstern, ibid. 101, 256802 (2008).

${ }^{10}$ R. J. Wagner, T. A. Kennedy, and B. D. McCombe, and D. C. Tsui, Phys. Rev. B 22, 945 (1980).

${ }^{11}$ The CR transmission line shape relates to the shape of LS's by the Fresnel formula. $^{12}$

${ }^{12}$ T. Ando, J. Phys. Soc. Jpn. 38, 989 (1975).

${ }^{13}$ M. L. Sadowski, G. Martinez, M. Potemski, C. Berger, and W. A. de Heer, Phys. Rev. Lett. 97, 266405 (2006).

${ }^{14}$ Z. Jiang, E. A. Henriksen, L. C. Tung, Y. -J. Wang, M. E. Schwartz, M. Y. Han, P. Kim, and H. L. Stormer, Phys. Rev. Lett. 98, 197403 (2007).
${ }^{15}$ M. Orlita, C. Faugeras, P. Plochocka, P. Neugebauer, G. Martinez, D. K. Maude, A. -L. Barra, M. Sprinkle, C. Berger, W. A. de Heer, and M. Potemski, Phys. Rev. Lett. 101, 267601 (2008).

${ }^{16}$ D. L. Miller, K. D. Kubista, G. M. Rutter, M. Ruan, W. A. de Heer, P. N. First, and J. A. Stroscio, Science 324, 924 (2009).

${ }^{17}$ D. L. Miller, K. D. Kubista, G. M. Rutter, M. Ruan, W. A. de Heer, M. Kindermann, P. N. First, and J. A. Stroscio, Nature Phys. 6, 811 (2010).

${ }^{18}$ G. Li and E. Y. Anderi, Nature Phys. 3, 623 (2007).

${ }^{19}$ G. Li, A. Luican, and E. Y. Andrei, Phys. Rev. Lett. 102, 176804 (2009).

${ }^{20}$ E. A. Henriksen and J. P. Eisenstein, Phys. Rev. B 82, 041412(R) (2010).

${ }^{21}$ L. A. Ponomarenko, R. Yang, R. V. Gorbachev, P. Blake, A. S. Mayorov, K. S. Novoselov, M. I. Katsnelson, and A. K. Geim, Phys. Rev. Lett. 105, 136801 (2010).

${ }^{22}$ F. Wegner, Z. Phys. B 51, 279 (1983).

${ }^{23}$ E. Brezin et al., Nucl. Phys. B 235, 24 (1984).

${ }^{24}$ W. Zhu, Q. W. Shi, X. R. Wang, J. Chen, J. L. Yang, and J. G. Hou, Phys. Rev. Lett. 102, 056803 (2009).

${ }^{25}$ T. Champel and S. Florens, Phys. Rev. B 82, 045421 (2010); 80, 161311(R) (2009).

${ }^{26}$ V. Krachmalnicoff, E. Castanie, Y. De Wilde, and R. Carminati, Phys. Rev. Lett. 105, 183901 (2010).

${ }^{27}$ X. R. Wang, Phys. Rev. B 51, 9310 (1995); 53, 12035 (1996).

${ }^{28}$ Y. Y. Zhang, J. P. Hu, B. A. Bernevig, X. R. Wang, X. C. Xie, and W. M. Liu, Phys. Rev. Lett. 102, 106401 (2009).

${ }^{29}$ The condition guarantees that the integral of impurity potential over the whole space does not depend on $d .^{30}$

${ }^{30}$ K. Wakabayashi, Y. Takane, and M. Sigrist, Phys. Rev. Lett. 99, 036601 (2007).

${ }^{31}$ S. D. Wu, L. Jing, Q. X. Li, Q. W. Shi, J. Chen, H. B. Su, X. P. Wang, and J. L. Yang, Phys. Rev. B 77, 195411 (2008).

${ }^{32}$ W. Zhu, Q. W. Shi, X. R. Wang, X. P. Wang, J. L. Yang, J. Chen, and J. G. Hou, Phys. Rev. B 82, 153405 (2010). 